Article

\title{
Preliminary Identification of Potential Vaccine Targets for the COVID-19 Coronavirus (SARS-CoV-2) Based on SARS-CoV Immunological Studies
}

\author{
Syed Faraz Ahmed ${ }^{1,+}+$ (i), Ahmed A. Quadeer ${ }^{1, *},+\infty$ and Matthew R. McKay ${ }^{1,2, *}$ \\ 1 Department of Electronic and Computer Engineering, The Hong Kong University of Science and Technology, \\ Hong Kong, China; sfahmed@connect.ust.hk \\ 2 Department of Chemical and Biological Engineering, The Hong Kong University of Science and Technology, \\ Hong Kong, China \\ * Correspondence: eeaaquadeer@ust.hk (A.A.Q); m.mckay@ust.hk (M.R.M.) \\ + These authors contributed equally to this work.
}

Received: 9 February 2020; Accepted: 24 February 2020; Published: 25 February 2020

\begin{abstract}
The beginning of 2020 has seen the emergence of COVID-19 outbreak caused by a novel coronavirus, Severe Acute Respiratory Syndrome Coronavirus 2 (SARS-CoV-2). There is an imminent need to better understand this new virus and to develop ways to control its spread. In this study, we sought to gain insights for vaccine design against SARS-CoV-2 by considering the high genetic similarity between SARS-CoV-2 and SARS-CoV, which caused the outbreak in 2003, and leveraging existing immunological studies of SARS-CoV. By screening the experimentally-determined SARS-CoV-derived B cell and T cell epitopes in the immunogenic structural proteins of SARS-CoV, we identified a set of $B$ cell and $T$ cell epitopes derived from the spike $(\mathrm{S})$ and nucleocapsid $(\mathrm{N})$ proteins that map identically to SARS-CoV-2 proteins. As no mutation has been observed in these identified epitopes among the 120 available SARS-CoV-2 sequences (as of 21 February 2020), immune targeting of these epitopes may potentially offer protection against this novel virus. For the $\mathrm{T}$ cell epitopes, we performed a population coverage analysis of the associated MHC alleles and proposed a set of epitopes that is estimated to provide broad coverage globally, as well as in China. Our findings provide a screened set of epitopes that can help guide experimental efforts towards the development of vaccines against SARS-CoV-2.
\end{abstract}

Keywords: Coronavirus; 2019-nCoV; 2019 novel coronavirus; SARS-CoV-2; COVID-19; SARS-CoV; MERS-CoV; T cell epitopes; B cell epitopes; vaccine

\section{Introduction}

The ongoing outbreak of COVID-19 in the Chinese city of Wuhan (Hubei province) [1] and its alarmingly quick transmission to 25 other countries across the world [2] resulted in the World Health Organization (WHO) declaring a global health emergency on 30 January 2020 [3]. This came just one month after the first reported case on 31 December 2019 [4]. WHO, in its first emergency meeting [5], estimated the fatality rate of COVID-19 to be around $4 \%$. Worldwide collaborative efforts from scientists are underway to understand the novel and rapidly spreading virus that causes this disease, SARS-CoV-2 (originally tentatively named 2019-nCoV), and to develop effective interventions for controlling and preventing it [6-9].

Coronaviruses are positive-sense single-stranded RNA viruses belonging to the family Coronaviridae. These viruses mostly infect animals, including birds and mammals. In humans, they generally cause mild respiratory infections, such as those observed in the common cold. However, some recent human coronavirus infections have resulted in lethal endemics, which include the SARS 
(Severe Acute Respiratory Syndrome) and MERS (Middle East Respiratory Syndrome) endemics. Both of these are caused by zoonotic coronaviruses that belong to the genus Betacoronavirus within Coronaviridae. SARS-CoV originated from Southern China and caused an endemic in 2003. A total of 8098 cases of SARS were reported globally, including 774 associated deaths, and an estimated case-fatality rate of 14\%-15\% [10]. The first case of MERS occurred in Saudi Arabia in 2012. Since then, a total of 2,494 cases of infection have been reported, including 858 associated deaths, and an estimated high case-fatality rate of $34.4 \%$ [11]. While no case of SARS-CoV infection has been reported since 2004, MERS-CoV has been around since 2012 and has caused multiple sporadic outbreaks in different countries.

Like SARS-CoV and MERS-CoV, the recent SARS-CoV-2 belongs to the Betacoronavirus genus [12]. It has a genome size of $\sim 30$ kilobases which, like other coronaviruses, encodes for multiple structural and non-structural proteins. The structural proteins include the spike $(S)$ protein, the envelope (E) protein, the membrane $(\mathrm{M})$ protein, and the nucleocapsid $(\mathrm{N})$ protein. With SARS-CoV-2 being discovered very recently, there is currently a lack of immunological information available about the virus (e.g., information about immunogenic epitopes eliciting antibody or $\mathrm{T}$ cell responses). Preliminary studies suggest that SARS-CoV-2 is quite similar to SARS-CoV based on the full-length genome phylogenetic analysis [9,12], and the putatively similar cell entry mechanism and human cell receptor usage $[9,13,14]$. Due to this apparent similarity between the two viruses, previous research that has provided an understanding of protective immune responses against SARS-CoV may potentially be leveraged to aid vaccine development for SARS-CoV-2.

Various reports related to SARS-CoV suggest a protective role of both humoral and cell-mediated immune responses. For the former case, antibody responses generated against the $S$ protein, the most exposed protein of SARS-CoV, have been shown to protect from infection in mouse models [15-17]. In addition, multiple studies have shown that antibodies generated against the N protein of SARS-CoV, a highly immunogenic and abundantly expressed protein during infection [18], were particularly prevalent in SARS-CoV-infected patients $[19,20]$. While being effective, the antibody response was found to be short-lived in convalescent SARS-CoV patients [21]. In contrast, $\mathrm{T}$ cell responses have been shown to provide long-term protection [21-23], even up to 11 years post-infection [24], and thus have also attracted interest for a prospective vaccine against SARS-CoV [reviewed in [25]]. Among all SARS-CoV proteins, $\mathrm{T}$ cell responses against the structural proteins have been found to be the most immunogenic in peripheral blood mononuclear cells of convalescent SARS-CoV patients as compared to the non-structural proteins [26]. Further, of the structural proteins, T cell responses against the $S$ and $\mathrm{N}$ proteins have been reported to be the most dominant and long-lasting [27].

Here, by analyzing available experimentally-determined SARS-CoV-derived B cell epitopes (both linear and discontinuous) and $\mathrm{T}$ cell epitopes, we identify and report those that are completely identical and comprise no mutation in the available SARS-CoV-2 sequences (as of 21 February 2020). These epitopes have the potential, therefore, to elicit a cross-reactive/effective response against SARS-CoV-2. We focused particularly on the epitopes in the $S$ and $N$ structural proteins due to their dominant and long-lasting immune response previously reported against SARS-CoV. For the identified T cell epitopes, we additionally incorporated the information about the associated MHC alleles to provide a list of epitopes that seek to maximize population coverage globally, as well as in China. Our presented results can potentially narrow down the search for potent targets for an effective vaccine against SARS-CoV-2, and help guide experimental studies focused on vaccine development.

\section{Materials and Methods}

\subsection{Acquisition and Processing of Sequence Data}

A total of 120 whole genome sequences of SARS-CoV-2 were downloaded on 21 February 2020 from the GISAID database (https://www.gisaid.org/CoV2020/) (Table S1). We excluded sequences that likely had spurious mutations resulting from sequencing errors, as indicated in the comment field 
of the GISAID data. These nucleotide sequences were aligned to the GenBank reference sequence (accession ID: NC_045512.2) and then translated into amino acid residues according to the coding sequence positions provided along the reference sequence for SARS-CoV-2 proteins (orf1a, orf1b, S, ORF3a, E, M, ORF6, ORF7a, ORF7b, ORF8, N, and ORF10). These sequences were aligned separately for each protein using the MAFFT multiple sequence alignment program [28]. Reference protein sequences for SARS-CoV and MERS-CoV were obtained following the same procedure from GenBank using the accession IDs NC_004718.3 and NC_019843.3, respectively.

\subsection{Acquisition and Filtering of Epitope Data}

SARS-CoV-derived B cell and T cell epitopes were searched on the NIAID Virus Pathogen Database and Analysis Resource (ViPR) (https://www.viprbrc.org/; accessed 21 February 2020) [29] by querying for the virus species name: "Severe acute respiratory syndrome-related coronavirus" from "human" hosts. We limited our search to include only the experimentally-determined epitopes that were associated with at least one positive assay: (i) Positive B cell assays (e.g., enzyme-linked immunosorbent assay (ELISA)-based qualitative binding) for B cell epitopes; and (ii) either positive $\mathrm{T}$ cell assays (such as enzyme-linked immune absorbent spot (ELISPOT) or intracellular cytokine staining (ICS) IFN- $\gamma$ release), or positive major histocompatibility complex (MHC) binding assays for T cell epitopes. Strictly speaking, the latter set of epitopes, determined using positive MHC binding assays, are antigens which are candidate epitopes, since a $\mathrm{T}$ cell response has not been confirmed experimentally. However, for brevity and to be consistent with the terminology used in the ViPR database, we will not make this qualification, and will simply refer to them as epitopes in this study. The number of $\mathrm{B}$ cell and $\mathrm{T}$ cell epitopes obtained from the database following the above procedure is listed in Table 1.

Table 1. Filtering criteria and corresponding number of Severe Acute Respiratory Syndrome Coronavirus (SARS-CoV)-derived epitopes obtained from the Virus Pathogen Database and Analysis Resource (ViPR) database.

\begin{tabular}{ccc}
\hline \multicolumn{2}{c}{ Filtering Criteria } & Number of Epitopes \\
\hline Positive T cell assays & T cell epitopes & 115 \\
\hline $\begin{array}{c}\text { Positive major histocompatibility } \\
\text { complex (MHC) binding assays }\end{array}$ & T cell epitopes & 959 \\
\hline \multirow{2}{*}{ Positive B cell assays } & Linear B cell epitopes & 298 \\
\cline { 2 - 3 } & Discontinuous B cell epitopes & 6 \\
\hline
\end{tabular}

\subsection{Population-Coverage-Based T Cell Epitope Selection}

Population coverages for sets of T cell epitopes were computed using the tool provided by the Immune Epitope Database (IEDB) (http://tools.iedb.org/population/; accessed 21 February 2020) [30]. This tool uses the distribution of MHC alleles (with at least 4-digit resolution, e.g., A*02:01) within a defined population (obtained from http:/www.allelefrequencies.net/) to estimate the population coverage for a set of $\mathrm{T}$ cell epitopes. The estimated population coverage represents the percentage of individuals within the population that are likely to elicit an immune response to at least one $\mathrm{T}$ cell epitope from the set. To identify the set of epitopes associated with MHC alleles that would maximize the population coverage, we adopted a greedy approach: (i) We first identified the MHC allele with the highest individual population coverage and initialized the set with their associated epitopes, then (ii) we progressively added epitopes associated with other MHC alleles that resulted in the largest increase of the accumulated population coverage. We stopped when no increase in the accumulated population coverage was observed by adding epitopes associated with any of the remaining MHC alleles. 


\subsection{Constructing the Phylogenetic Tree}

We used the publicly available software PASTA v1.6.4 [31] to construct a maximum-likelihood phylogenetic tree of each structural protein using the unique set of sequences in the available data of SARS-CoV, MERS-CoV, and SARS-CoV-2. We additionally included the Zaria Bat coronavirus strain (accession ID: HQ166910.1) to serve as an outgroup. The appropriate parameters for tree estimation are automatically selected in the software based on the provided sequence data. For visualizing the constructed phylogenetic trees, we used the publicly available software Dendroscope v3.6.3 [32]. Each constructed tree was rooted with the outgroup Zaria Bat coronavirus strain, and circular phylogram layout was used.

\subsection{Data and Code Availability}

All sequence and immunological data, and all scripts (written in R) for reproducing the results are available online [33].

\section{Results}

\subsection{Structural Proteins of SARS-CoV-2 Are Genetically Similar to SARS-CoV, but Not to MERS-CoV}

SARS-CoV-2 has been observed to be close to SARS-CoV-much more so than MERS-CoV-based on full-length genome phylogenetic analysis [9,12]. We checked whether this is also true at the level of the individual structural proteins ( $, E, M$, and N). A straightforward reference-sequence-based comparison indeed confirmed this, showing that the $M, N$, and E proteins of SARS-CoV-2 and SARS-CoV have over $90 \%$ genetic similarity, while that of the $S$ protein was notably reduced (but still high) (Figure 1a). The similarity between SARS-CoV-2 and MERS-CoV, on the other hand, was substantially lower for all proteins (Figure 1a); a feature that was also evident from the corresponding phylogenetic trees (Figure 1b). We note that while the former analysis (Figure 1a) was based on the reference sequence of each coronavirus, it is indeed a good representative of the virus population, since few amino acid mutations have been observed in the corresponding sequence data (Figure S1). It is also noteworthy that while MERS-CoV is the more recent coronavirus to have infected humans, and is comparatively more recurrent (causing outbreaks in 2012, 2015, and 2018) (https://www.who.int/emergencies/mers-cov/en/), SARS-CoV-2 is closer to SARS-CoV, which has not been observed since 2004.

a

Percentage sequence identity with SARS-CoV-2

\begin{tabular}{|lllll|}
\hline & S protein & N protein & M protein & E protein \\
\hline SARS-CoV & $76.0 \%$ & $90.6 \%$ & $90.1 \%$ & $94.7 \%$ \\
\hline MERS-CoV & $29.4 \%$ & $45.9 \%$ & $39.2 \%$ & $34.1 \%$ \\
\hline
\end{tabular}

b

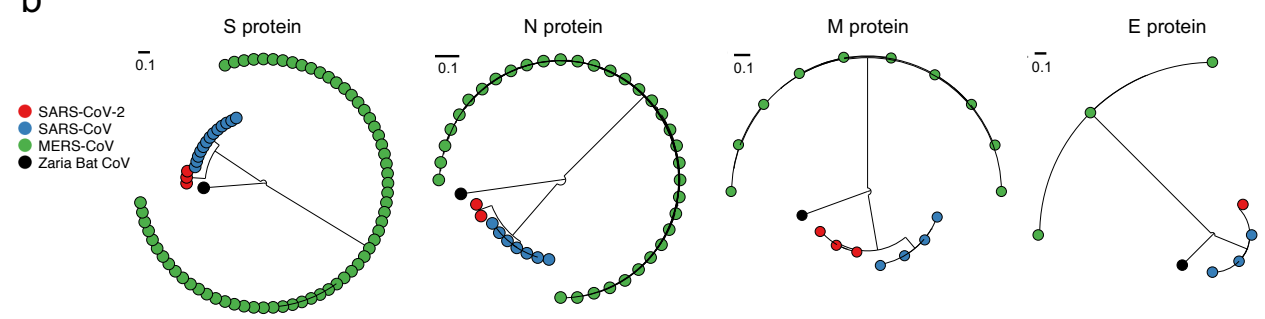

Figure 1. Comparison of the similarity of structural proteins of SARS-CoV-2 with the corresponding proteins of SARS-CoV and MERS (Middle East Respiratory Syndrome)-CoV. (a) Percentage genetic similarity of the individual structural proteins of SARS-CoV-2 with those of SARS-CoV and MERS-CoV. The reference sequence of each coronavirus (Materials and Methods) was used to calculate the percentage genetic similarity. (b) Circular phylogram of the phylogenetic trees of the four structural proteins. All trees were constructed based on the available unique sequences using PASTA [31] and rooted with the outgroup Zaria Bat CoV strain (accession ID: HQ166910.1). 
Given the close genetic similarity between the structural proteins of SARS-CoV and SARS-CoV-2, we attempted to leverage immunological studies of the structural proteins of SARS-CoV to potentially aid vaccine development for SARS-CoV-2. We focused specifically on the $\mathrm{S}$ and $\mathrm{N}$ proteins as these are known to induce potent and long-lived immune responses in SARS-CoV [15-17,19,20,25,27]. We used the available SARS-CoV-derived experimentally-determined epitope data (see Materials and Methods) and searched to identify $\mathrm{T}$ cell and $\mathrm{B}$ cell epitopes that were identical—and hence potentially cross-reactive-across SARS-CoV and SARS-CoV-2. We first report the analysis for T cell epitopes, which have been shown to provide a long-lasting immune response against SARS-CoV [27], followed by a discussion of $B$ cell epitopes.

\subsection{Mapping the SARS-CoV-Derived T Cell Epitopes That Are Identical in SARS-CoV-2, and Determining Those with Greatest Estimated Population Coverage}

The SARS-CoV-derived T cell epitopes used in this study were experimentally-determined from two different types of assays [29]: (i) Positive T cell assays, which tested for a $\mathrm{T}$ cell response against epitopes, and (ii) positive MHC binding assays, which tested for epitope-MHC binding. We aligned these $\mathrm{T}$ cell epitopes across the SARS-CoV-2 protein sequences. Among the $115 \mathrm{~T}$ cell epitopes that were determined by positive $\mathrm{T}$ cell assays (Table 1 ), we found that 27 epitope-sequences were identical within SARS-CoV-2 proteins and comprised no mutation in the available SARS-CoV-2 sequences (as of 21 February 2020) (Table 2). Interestingly, all of these were present in either the N (16) or S (11) protein. MHC binding assays were performed for 19 of these 27 epitopes, and these were reported to be associated with only five distinct MHC alleles (at 4-digit resolution): HLA-A*02:01, HLA-B*40:01, HLA-DRA*01:01, HLA-DRB1*07:01, and HLA-DRB1*04:01. Consequently, the accumulated population coverage of these epitopes (see Materials and Methods for details) is estimated to not be high for the global population (59.76\%), and was quite low for China (32.36\%). For the remaining 8 epitopes, since the associated MHC alleles are unknown, they could not be used in the population coverage computation. Additional MHC binding tests to identify the MHC alleles that bind to these 8 epitopes may reveal additional distinct alleles, beyond the five determined so far, that may help to improve population coverage.

Table 2. SARS-CoV-derived $\mathrm{T}$ cell epitopes obtained using positive $\mathrm{T}$ cell assays that are identical in SARS-CoV-2 (27 epitopes in total).

\begin{tabular}{|c|c|c|c|c|}
\hline Protein & IEDB ID & Epitope & MHC Allele ${ }^{1}$ & MHC Allele Class ${ }^{1}$ \\
\hline $\mathrm{N}$ & 125100 & ILLNKHID & HLA-A*02:01 & $\mathrm{I}$ \\
\hline $\mathrm{N}$ & 1295 & AFFGMSRIGMEVTPSGTW & NA & NA \\
\hline $\mathrm{N}$ & 190494 & MEVTPSGTWL & HLA-B*40:01 & $\mathrm{I}$ \\
\hline $\mathrm{N}$ & 21347 & GMSRIGMEV & HLA-A*02:01 & I \\
\hline $\mathrm{N}$ & 27182 & ILLNKHIDA & HLA-A*02:01 & $\mathrm{I}$ \\
\hline $\mathrm{N}$ & 2802 & ALNTPKDHI & HLA-A*02:01 & I \\
\hline $\mathrm{N}$ & 28371 & IRQGTDYKHWPQIAQFA & NA & NA \\
\hline $\mathrm{N}$ & 31166 & KHWPQIAQFAPSASAFF & NA & NA \\
\hline $\mathrm{N}$ & 34851 & LALLLLDRL & HLA-A*02:01 & I \\
\hline $\mathrm{N}$ & 37473 & LLLDRLNQL & HLA-A*02:01 & $\mathrm{I}$ \\
\hline $\mathrm{N}$ & 37611 & LLNKHIDAYKTFPPTEPK & NA & NA \\
\hline $\mathrm{N}$ & 38881 & LQLPQGTTL & HLA-A*02:01 & $\mathrm{I}$ \\
\hline
\end{tabular}


Table 2. Cont.

\begin{tabular}{ccccc}
\hline Protein & IEDB ID & Epitope & MHC Allele & MHC Allele Class \\
\hline N & 3957 & AQFAPSASAFFGMSR & NA & II \\
\hline N & 3958 & AQFAPSASAFFGMSRIGM & NA & NA \\
\hline N & 55683 & RRPQGLPNNTASWFT & NA & NA \\
\hline N & 74517 & YKTFPPTEPKKDKKKK & NA & II \\
\hline S & 100048 & GAALQIPFAMQMAYRF & HLA-DRA*01:01, & HLA-DRB1*07:01 \\
\hline S & 100300 & MAYRFNGIGVTQNVLY & HLA-DRB1*04:01 & II \\
\hline S & 100428 & QLIRAAEIRASANLAATK & HLA-DRB1*04:01 & I \\
\hline S & 16156 & FIAGLIAIV & HLA-A*02:01 & I \\
\hline S & 2801 & ALNTLVKQL & HLA-A*02:01 & I \\
\hline S & 36724 & LITGRLQSL & HLA-A2 & I \\
\hline S & 44814 & NLNESLIDL & HLA-A*02:01 & II \\
\hline S & 50311 & QALNTLVKQLSSNFGAI & HLA-DRB1*04:01 & I \\
\hline S & 54680 & RLNEVAKNL & HLA-A*02:01 & I \\
\hline S & 69657 & VLNDILSRL & HLA-A*02:01 & I \\
\hline S & 71663 & VVFLHVTYV & HLA-A*02:01 & \\
\hline
\end{tabular}

To further expand the search and identify potentially effective $T$ cell targets covering a higher percentage of the population, we next additionally considered the set of $\mathrm{T}$ cell epitopes that have been experimentally-determined from positive MHC binding assays (Table 1), but, unlike the previous epitope set, their ability to induce a $\mathrm{T}$ cell response against SARS-CoV was not experimentally determined. Nonetheless, they also present promising candidates for inducing a response against SARS-CoV-2. For the expanded set of epitopes, all of which have at least one positive MHC binding assay, we found that 229 epitope-sequences have an identical match in SARS-CoV-2 proteins and have associated MHC allele information available (listed in Table S2). Of these 229 epitopes, $\sim 82 \%$ were MHC Class I restricted epitopes (Table S3). Importantly, 102 of the 229 epitopes were derived from either the S (66) or N (36) protein. Mapping all 66 S-derived epitopes onto the resolved crystal structure of the SARS-CoV S protein (Figure S2) revealed that 3 of these (GYQPYRVVVL, QPYRVVVLSF, and PYRVVVLSF) were located entirely in the SARS-CoV receptor-binding motif (https://www.uniprot.org/uniprot/P59594), known to be important for virus cell entry [34].

Similar to previous studies on HIV and HCV [35-38], we estimated population coverages for various combinations of MHC alleles associated with these 102 epitopes. Our aim was to determine sets of epitopes associated with MHC alleles with maximum population coverage, potentially aiding the development of vaccines against SARS-CoV-2. For selection, we adopted a greedy computational approach (see Materials and Methods), which identified a set of $\mathrm{T}$ cell epitopes estimated to maximize global population coverage. This set comprised of multiple $T$ cell epitopes associated with 20 distinct MHC alleles and was estimated to provide an accumulated population coverage of $96.29 \%$ (Table 3). Interestingly, the majority of the $\mathrm{T}$ cell epitopes for which a positive immune response has been determined using T cell assays (Table 2) were presented by the globally most-prevalent MHC allele (shown in blue color in Table 3). Moreover, the functionally important epitopes located in the SARS-CoV receptor binding motif were associated with the second and third most-prevalent MHC alleles (underlined in Table 3). Thus, while the ordering of T cell epitopes in Table 3 is based on the estimated global population coverage of the associated MHC alleles, it is also a natural order in which these epitopes should be tested experimentally for determining their potential to induce a positive immune response against SARS-CoV-2. We also computed the population coverage of this specific set 
of epitopes in China, the country most affected by the COVID-19 outbreak, which was estimated to be slightly lower $(88.11 \%$ ), as certain MHC alleles (e.g., HLA-A*02:01) associated with some of these epitopes are less frequent in the Chinese population (Table 3). Repeating the same greedy approach but focusing on the Chinese population, instead of a global population, the maximum population coverage was estimated to be $92.76 \%$ (Table S4).

Table 3. Set of the SARS-CoV-derived spike (S) and nucleocapsid (N) protein T cell epitopes (obtained from positive MHC binding assays) that are identical in SARS-CoV-2 and that maximize estimated population coverage globally (87 distinct epitopes).

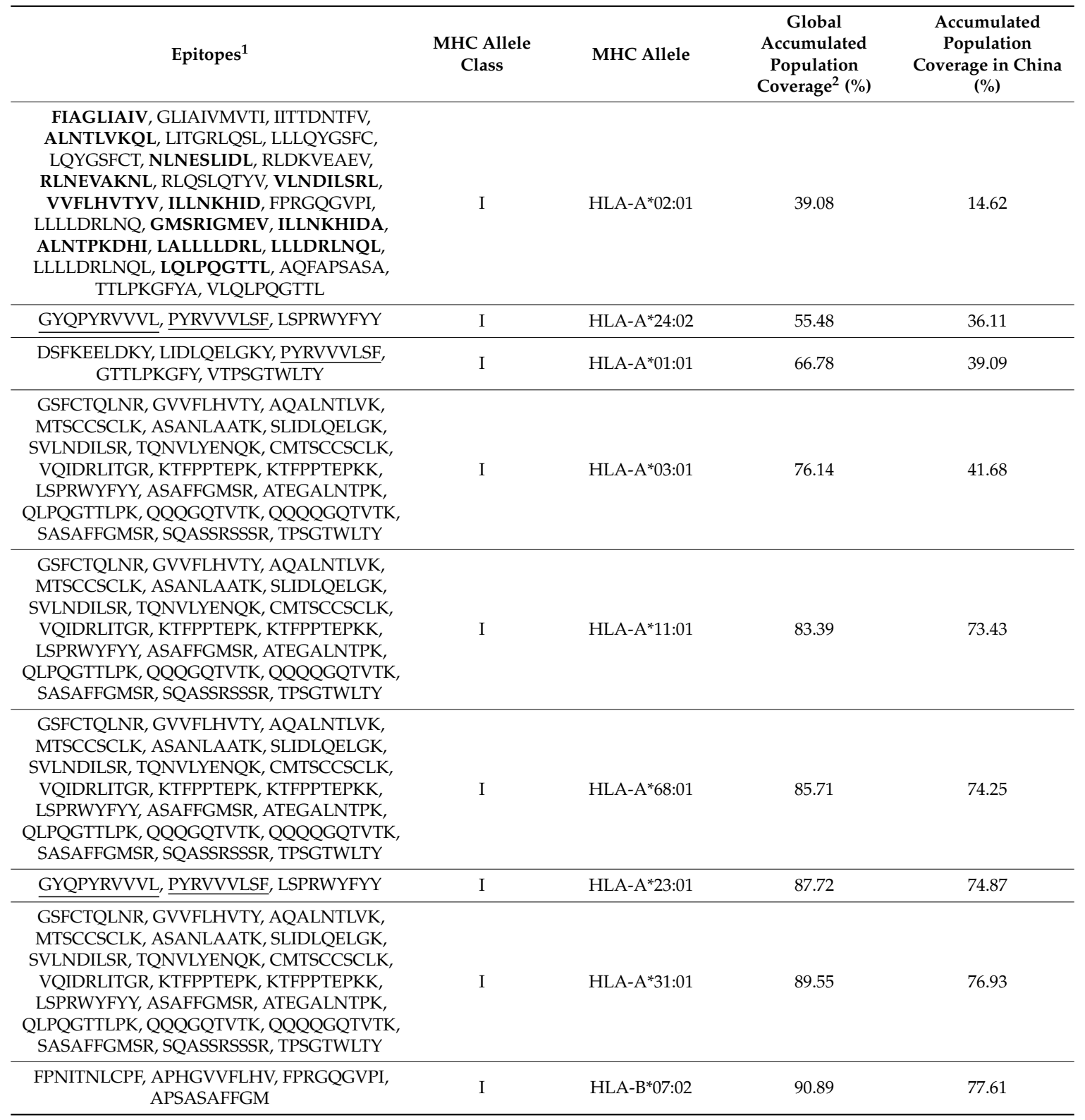


Table 3. Cont.

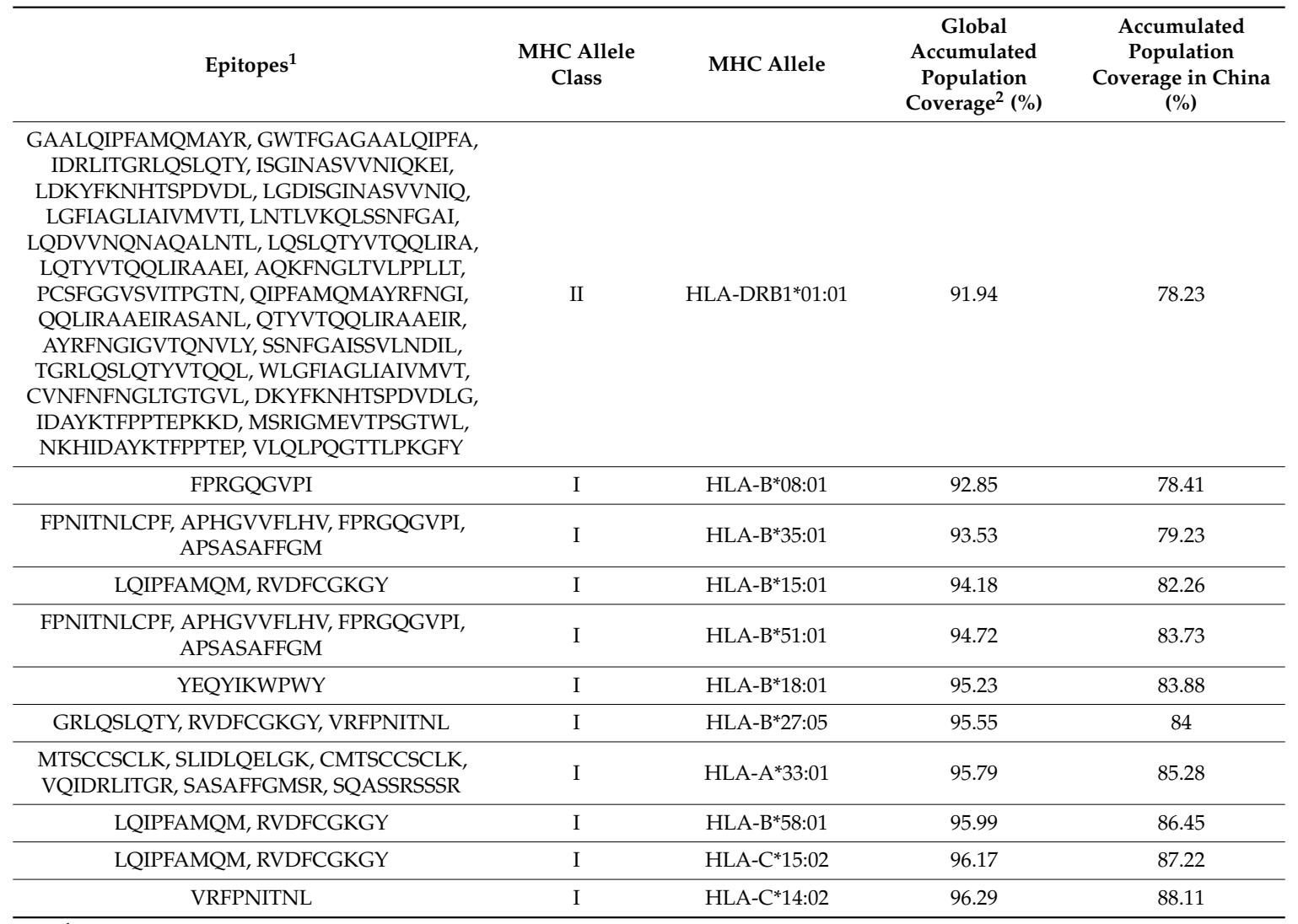

${ }^{1}$ Multiple SARS-CoV-derived epitopes that were determined using MHC binding assays are shown for each allele. Epitopes that were also tested for positive T cell response (listed also in Table 2) are shown in bold. Epitopes that lie within the SARS-CoV receptor-binding motif are underlined. ${ }^{2}$ Epitopes are ordered according to the estimated global accumulated population coverage.

Due to the promiscuous nature of binding between peptides and MHC alleles, multiple $S$ and $\mathrm{N}$ peptides were reported to bind to individual MHC alleles. Thus, while we list all the $\mathrm{S}$ and $\mathrm{N}$ epitopes that bind to each MHC allele (Table 3), the estimated maximum population coverage may be achieved by selecting at least one epitope for each listed MHC allele. Likewise, many individual S and $\mathrm{N}$ epitopes were found to be presented by multiple alleles and thereby estimated to have varying global population coverage (listed in Table S5).

\subsection{Mapping the SARS-CoV-Derived B cell Epitopes that Are Identical in SARS-CoV-2}

Similar to T cell epitopes, we used in our study the SARS-CoV-derived B cell epitopes that have been experimentally-determined from positive B cell assays [29]. These epitopes were classified as: (i) Linear B cell epitopes (antigenic peptides), and (ii) discontinuous B cell epitopes (conformational epitopes with resolved structural determinants).

We aligned the 298 linear B cell epitopes (Table 1) across the SARS-CoV-2 proteins and found that 49 epitope-sequences, all derived from structural proteins, have an identical match and comprised no mutation in the available SARS-CoV-2 protein sequences (as of 21 February 2020). Interestingly, a large number (45) of these were derived from either the $S$ (23) or N (22) protein (Table 4), while the remaining (4) were from the M protein (Table S6). 
Table 4. SARS-CoV-derived linear B cell epitopes from S (23; 20 of which are located in subunit S2) and $\mathrm{N}$ (22) proteins that are identical in SARS-CoV-2 (45 epitopes in total).

\begin{tabular}{|c|c|c|c|c|c|c|}
\hline Protein & Subunit & IEDB ID & Epitope & Protein & IEDB ID & Epitope \\
\hline $\mathrm{s}$ & S2 & 10778 & DVVNQNAQALNTLVKQL & $\mathrm{N}$ & 15814 & FFGMSRIGMEVTPSGTW \\
\hline $\mathrm{s}$ & S2 & 11038 & EAEVQIDRLITGRLQSL & $\mathrm{N}$ & 21065 & GLPNNTASWFTALTQHGK \\
\hline $\mathrm{S}$ & S2 & 12426 & EIDRLNEVAKNLNESLIDLQELGKYEQY & $\mathrm{N}$ & 22855 & GTTLPK \\
\hline $\mathrm{S}$ & S2 & 14626 & EVAKNLNESLIDLQELG & $\mathrm{N}$ & 28371 & IRQGTDYKHWPQIAQFA \\
\hline $\mathrm{s}$ & S2 & 18515 & GAALQIPFAMQMAYRFN & $\mathrm{N}$ & 31116 & KHIDAYKTFPРTEPKKDKKK \\
\hline $\mathrm{s}$ & S1 & 18594 & GAGICASY & $\mathrm{N}$ & 31166 & KHWPQIAQFAPSASAFF \\
\hline $\mathrm{s}$ & S2 & 2092 & AISSVLNDILSRLDKVE & $\mathrm{N}$ & 75235 & YNVTQAFGRRGPEQTQGNF \\
\hline $\mathrm{s}$ & S2 & 22321 & GSFCTQLN & $\mathrm{N}$ & 33669 & КTFPРTEPKKDKKKK \\
\hline $\mathrm{s}$ & S2 & 27357 & ILSRLDKVEAEVQIDRL & $\mathrm{N}$ & 37640 & LLPAAD \\
\hline $\mathrm{S}$ & S1 & 30987 & KGIYQTSN & $\mathrm{N}$ & 38249 & LNKHIDAYKTFPPTEPK \\
\hline $\mathrm{s}$ & S2 & 3176 & AMQMAYRF & $\mathrm{N}$ & 38648 & LPQGTTLPKG \\
\hline $\mathrm{S}$ & S2 & 32508 & KNHTSPDVDLGDISGIN & $\mathrm{N}$ & 38657 & LPQRQKKQ \\
\hline $\mathrm{S}$ & S2 & 41177 & MAYRFNGIGVTQNVLYE & $\mathrm{N}$ & 48067 & PKGFYAEGSRGGSQASSR \\
\hline $\mathrm{s}$ & S2 & 462 & AATKMSECVLGQSKRVD & $\mathrm{N}$ & 50741 & QFAPSASAFFGMSRIGM \\
\hline $\mathrm{s}$ & S2 & 47479 & PFAMQMAYRFNGIGVTQ & $\mathrm{N}$ & 50965 & QGTDYKHW \\
\hline $\mathrm{s}$ & S2 & 50311 & QALNTLVKQLSSNFGAI & $\mathrm{N}$ & 51483 & QLPQGTTLPKGFYAE \\
\hline $\mathrm{s}$ & S2 & 51379 & QLIRAAEIRASANLAAT & $\mathrm{N}$ & 51484 & QLPQGTTLPKGFYAEGSR \\
\hline $\mathrm{s}$ & S1 & 52020 & QQFGRD & $\mathrm{N}$ & 51485 & QLPQGTTLPKGFYAEGSRGGSQ \\
\hline $\mathrm{s}$ & S2 & 53202 & RASANLAATKMSECVLG & $\mathrm{N}$ & 63729 & TFPPTEPK \\
\hline $\mathrm{s}$ & S2 & 54599 & RLITGRLQSLQTYVTQQ & $\mathrm{N}$ & 55683 & RRPQGLPNNTASWFT \\
\hline $\mathrm{s}$ & S2 & 558417 & EIDRLNEVAKNLNESLIDLQELGKYEQY & $\mathrm{N}$ & 60379 & SQASSRSS \\
\hline $\mathrm{S}$ & S2 & 59425 & SLQTYVTQQLIRAAEIR & $\mathrm{N}$ & 60669 & SRGGSQASSRSSSRSR \\
\hline $\mathrm{s}$ & S2 & 9094 & DLGDISGINASVVNIQK & & & \\
\hline
\end{tabular}

On the other hand, all 6 SARS-CoV-derived discontinuous B cell epitopes obtained from the ViPR database (Table 5) were derived from the $S$ protein. Based on the pairwise alignment between the SARS-CoV and SARS-CoV-2 reference sequences (Figure S3), we found that none of these mapped identically to the SARS-CoV-2 S protein, in contrast to the linear epitopes. For 3 of these discontinuous B cell epitopes (corresponding to antibodies S230, m396, and 80R [39-41]), there was a partial mapping, with at least one site having an identical residue at the corresponding site in the SARS-CoV-2 S protein (Table 5).

Table 5. SARS-CoV-derived discontinuous B cell epitopes (and associated known antibodies [39-41]) that have at least one site with an identical amino acid to the corresponding site in SARS-CoV-2.

\begin{tabular}{|c|c|c|}
\hline IEDB ID & Associated Known Antibody & SARS-CoV S Protein Residues ${ }^{1,2}$ \\
\hline 910052 & S230 & G446, P462, D463, $\underline{\mathrm{Y} 475}$ \\
\hline 77444 & $\mathrm{~m} 396$ & 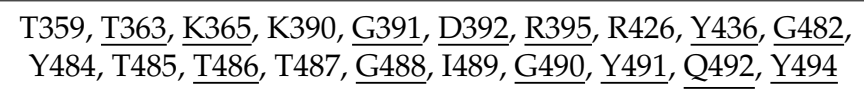 \\
\hline 77442 & $80 \mathrm{R}$ & $\begin{array}{l}\text { R426, S432, T433, Y436, N437, K439, Y440, Y442, P469, P470, } \\
\text { A471, L472, N473, } \underline{\mathrm{C} 474}, \underline{\mathrm{Y} 475}, \mathrm{~W} 476, \underline{\mathrm{L} 478}, \mathrm{~N} 479, \mathrm{D} 480, \underline{\mathrm{Y} 481}, \\
\mathrm{G} 482, \mathrm{Y} 484, \mathrm{~T} 485, \underline{\mathrm{T}} 486, \mathrm{~T} 487, \mathrm{G} 488, \underline{\mathrm{I} 489}, \underline{\mathrm{Y} 491}, \mathrm{Q} 492\end{array}$ \\
\hline
\end{tabular}

${ }^{1}$ Residues are numbered according to the SARS-CoV S protein reference sequence, accession ID: NP_828851.1.; ${ }^{2}$ Residues in the epitopes that are identical in the SARS-CoV-2 sequences are underlined.

Mapping the residues of the linear and discontinuous B cell epitopes onto the available structure of the SARS-CoV S protein revealed their distinct association with the two functional subunits of the $S$ protein [42]: S1, important for interaction with the host cell receptor, and S2, involved in fusion of the cellular and virus membranes (Figure 2a). Specifically, 20 of the 23 linear epitopes (Table 4) mapped to S2 (Figure 2b). Thus, the antibodies targeting the identified linear epitopes in the S2 subunit might cross-react and neutralize both SARS-CoV and SARS-CoV-2, as suggested in a very recent study [43]. 
While S2 is comparatively less exposed than S1, it may be accessible to antibodies during the complex conformational changes involved in viral entry of coronaviruses [44-46]; though this remains to be more clearly understood. In contrast, the 3 discontinuous B cell epitopes (Table 5) mapped onto the more exposed S1 subunit (Figure 2c, left panel), which contains the receptor-binding motif of the SARS-CoV S protein [34]. We observed that very few residues of the 3 discontinuous epitopes were identical within SARS-CoV and SARS-CoV-2 (Figure 2c, right panel). These differences suggest that the SARS-CoV-specific antibodies S230, m396, and 80R known to bind to these epitopes in SARS-CoV might not be able to bind to the same regions in SARS-CoV-2 S protein. Interestingly, while this paper was under review, this has been confirmed experimentally [47]. Further studies are currently under way to identify other SARS-CoV antibodies that may bind to discontinuous epitopes of the SARS-CoV-2 $\mathrm{S}$ protein [48].

a

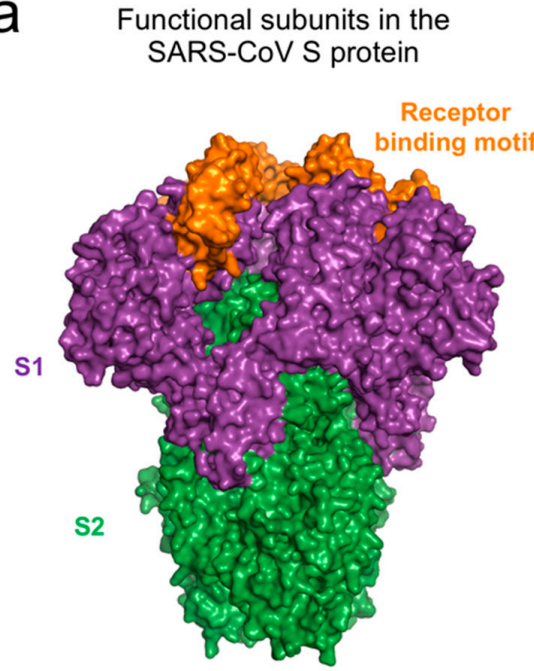

C

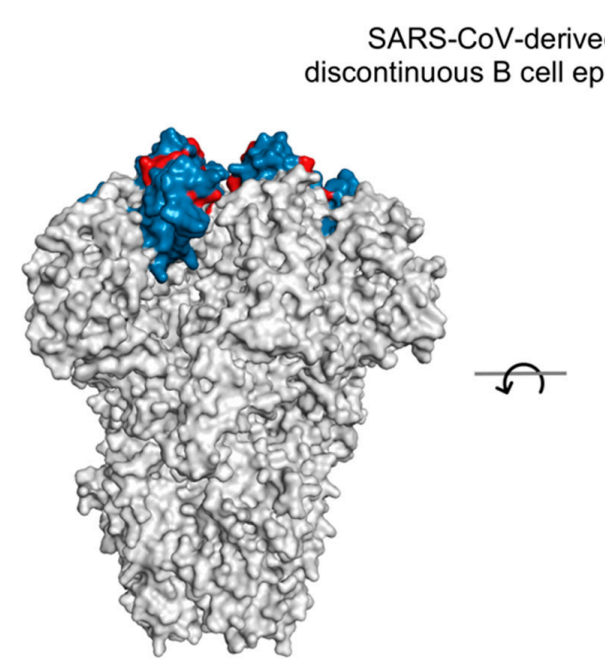

b SARS-CoV-derived linear $\mathrm{B}$ cell epitopes (selected)

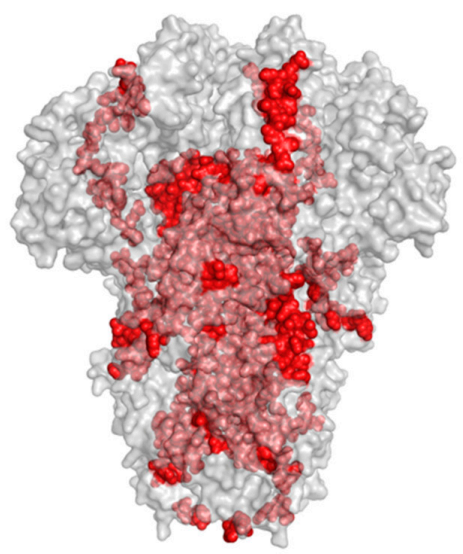

Identical residues in SARS-CoV-2 Non-identical residues in SARS-CoV-2

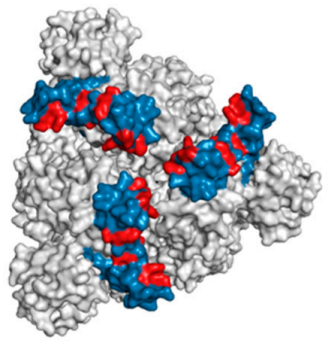

Figure 2. Location of SARS-CoV S protein subunits and SARS-CoV-derived B cell epitopes on the protein structure (PDB ID: 5XLR). (a) Subunits S1 and S2 are indicated in purple and green color, respectively. The receptor binding motif lies within the S1 subunit and is indicated in orange color. (b) Residues of the linear B cell epitopes, that were identical in SARS-CoV-2 (Table 4), are shown in red color. The dark and light shade reflect the surface and buried residues, respectively. (c) Location of discontinuous B cell epitopes that share at least one identical residue with corresponding SARS-CoV-2 sites (Table 5). Identical epitope residues are shown in red color, while the remaining epitope residues are shown in blue color. Both the side view (left panel) and the top view (right panel) of the structure are shown. 


\section{Discussion}

The quest for a vaccine against the novel SARS-CoV-2 is recognized as an urgent problem. Effective vaccination could indeed play a significant role in curbing the spread of the virus, and help to eliminate it from the human population. However, scientific efforts to address this challenge are only just beginning. Much remains to be learnt about the virus, its biological properties, epidemiology, etc. At this early stage, there is also a lack of information about specific immune responses against SARS-CoV-2, which presents a challenge for vaccine development.

This study has sought to assist with the initial phase of vaccine development by providing recommendations of epitopes that may potentially be considered for incorporation in vaccine designs. Despite having limited understanding of how the human immune system responds naturally to SARS-CoV-2, these epitopes are motivated by responses they have recorded in SARS-CoV (or, for the case of $\mathrm{T}$ cell epitopes, to at least confer MHC binding), and the fact that they map identically to SARS-CoV-2, based on the available sequence data (as of 21 February 2020). This important observation should not be taken for granted. Despite the apparent similarity between SARS-CoV and SARS-CoV-2, there is still considerable genetic variation between the two, and it is not obvious a-prior if epitopes that elicit an immune response against SARS-CoV are likely to be effective against SARS-CoV-2. We found that only $23 \%$ and $16 \%$ of known SARS-CoV $\mathrm{T}$ cell and B cell epitopes map identically to SARS-CoV-2, respectively, and with no mutation having been observed in these epitopes among the available SARS-CoV-2 sequences (as of 21 February 2020). This provides a strong indication of their potential for eliciting a robust $\mathrm{T}$ cell or antibody response in SARS-CoV-2.

On the $\mathrm{T}$ cell side, the identification of SARS-CoV-derived epitopes that map identically to SARS-CoV-2, and the large population that these are expected to cover, is particularly encouraging. It promotes further research in exploring vaccines designed to induce a protective $\mathrm{T}$ cell response, which has been shown to provide long term protection in SARS-CoV [21-24]. On the B cell side, in agreement with very recent experimental studies $[47,48]$, our results suggest that SARS-CoV-derived antibodies targeting the receptor binding motif in the S1 subunit of the SARS-CoV-2 S protein may not be effective, due to the large genetic mismatches observed in known structural epitopes targeting this domain. Linear SARS-CoV-derived B cell epitopes in the S2 subunit may potentially be more promising candidates for inducing a protective antibody response. Numerous of these epitopes, while being less exposed, are found to map identically to SARS-CoV and SARS-CoV-2, and preliminary results are already emerging which suggest their potential in generating cross-reactive and neutralizing antibodies [43]. Hence, vaccine solutions that attempt to induce antibodies that target the S2 linear epitopes may be effective and should be explored further.

Research efforts directed towards the design and development of vaccines for SARS-CoV-2 are increasing, and some related analyses are already being reported in distinct, parallel studies. These studies, like our own, are based on leveraging available data and computational methods, and add to recent work focused on computational analysis and design of vaccines for various different viruses (e.g., [49-54]. A preliminary analysis of linear SARS-CoV-derived B cell epitopes has been reported online on the ViPR database website (https://www.viprbrc.org/brcDocs/documents/announcements/ Corona/2019-nCoV-ViPR-report_24JAN2020.pdf). Different from our study, which is focused on the linear and discontinuous SARS-CoV-derived epitopes, that analysis considered linear B cell epitope data for all Betacoronaviruses from human hosts. While only a summary of the results has been provided so far, preventing direct comparison of the individual epitopes, the number of linear B cell epitopes reported to map identically to SARS-CoV-2 is comparable to our findings.

A recent study has also predicted $\mathrm{T}$ cell epitopes for SARS-CoV-2 that may be presented by a population from the Asia-Pacific region [55]. Again, there are multiple differences to our work. First, the focus of that study was on MHC Class II epitopes, while here we considered both MHC Class I and II epitopes. Interestingly, while we found a few MHC Class II epitopes using our approach (Table S3), only one of these (HLA-DRB1*01:01) appeared in our identified epitope set (Table 3), due to their comparatively low estimated population coverage. Second, computational tools were used to predict 
MHC Class II epitopes in [55], while here we analyzed the SARS-CoV-derived epitopes that have been determined experimentally, using either positive $\mathrm{T}$ cell or MHC binding assays, and which match identically with the available SARS-CoV-2 sequences (as of 21 February 2020). Thus, our identified epitopes are seemingly a more rational set of potential targets that can assist in the ongoing search for a SARS-CoV-2 vaccine.

We acknowledge that this is a preliminary analysis based on the limited sequence data available for SARS-CoV-2 (as of 21 February 2020). As the virus continues to evolve and as more data is collected, it is expected that additional mutations will be observed. Such mutations will not affect our analysis, provided that they occur outside of the identified epitope regions. If mutations do occur within epitope regions, then these epitopes may be further screened in line with the conservative filtering principle that we have employed, thereby producing a more refined epitope set.

Further experimental studies ( $\mathrm{T}$ cell and B cell assays) are required to determine the potential of the identified epitopes to induce a positive immune response against SARS-CoV-2. This would help to further refine the reported epitope set, based on observed immunogenicity; an important consideration for immunogen design.

Overall, as the identified set of SARS-CoV epitopes map identically to SARS-CoV-2, they present potentially useful candidates for guiding experimental efforts towards developing vaccines against SARS-CoV-2. More generally, our study further highlights the potential importance of previous experimental and clinical studies of SARS-CoV, and its use in concert with emerging data for SARS-CoV-2, in searching for effective vaccines to combat the COVID-19 epidemic.

Supplementary Materials: The following are available online at http://www.mdpi.com/1999-4915/12/3/254/s1, Figure S1: Fraction of mutations in the observed sequences of the structural proteins of the three coronaviruses, Figure S2: Location of identified T cell epitopes on the SARS-CoV S protein structure (PDB ID: 5XLR), Figure S3: Pairwise sequence alignment of the reference sequences of the S proteins of SARS-CoV and SARS-CoV-2 (accession ID: NP_828851.1 and YP_009724390.1, respectively), Table S1: List of GISAID accession IDs for 120 genomic sequences of SARS-CoV-2, Table S2: List of all SARS-CoV-derived T cell epitopes determined using positive MHC binding assays (with associated MHC allele information available at 4 digit resolution) and found to be identical in SARS-CoV-2, Table S3: Distribution of all SARS-CoV-derived T cell epitopes obtained using positive MHC binding assays (with associated MHC allele information available at 4 digit resolution) and that are identical in SARS-CoV-2, Table S4: Set of SARS-CoV-derived S and N protein T cell epitopes (obtained using positive MHC binding assays) that are identical in SARS-CoV-2 and that maximize estimated population coverage in China (86 distinct epitopes), Table S5: Estimated global and Chinese population coverages for the individual SARS-CoV-derived S or N protein T cell epitopes (obtained using positive MHC binding assays), that are identical in SARS-CoV-2, Table S6: SARS-CoV-derived linear B cell epitopes, excluding those in S and N proteins, that are identical in SARS-CoV-2, Table S7: Acknowledgment table.

Author Contributions: Conceptualization: S.F.A., A.A.Q., and M.R.M.; methodology: S.F.A., A.A.Q., and M.R.M.; software: S.F.A. and A.A.Q.; validation: S.F.A. and A.A.Q.; formal analysis: S.F.A., A.A.Q., and M.R.M.; investigation: S.F.A., A.A.Q., and M.R.M.; resources: M.R.M.; data curation: S.F.A.; writing-original draft preparation: S.F.A., A.A.Q., and M.R.M.; writing-review and editing: S.F.A., A.A.Q., and M.R.M.; visualization: S.F.A. and A.A.Q.; supervision: A.A.Q. and M.R.M.; project administration: A.A.Q. and M.R.M.; funding acquisition: M.R.M. All authors have read and agreed to the published version of the manuscript.

Acknowledgments: We thank all the authors, the originating and submitting laboratories (listed in Table S7) for their sequence and metadata shared through GISAID, on which this research is based. M.R.M. and A.A.Q. were supported by the General Research Fund of the Hong Kong Research Grants Council (RGC) [Grant No. 16204519]. S.F.A. was supported by the Hong Kong Ph.D. Fellowship Scheme (HKPFS).

Conflicts of Interest: The authors declare no conflict of interest.

\section{References}

1. Wang, C.; Horby, P.W.; Hayden, F.G.; Gao, G.F. A novel coronavirus outbreak of global health concern. Lancet 2020, 395, 470-473. [CrossRef]

2. Centers-of-Disease-Control-and-Prevention Confirmed 2019-nCoV cases globally. Available online: https: //www.cdc.gov/coronavirus/2019-ncov/locations-confirmed-cases.html (accessed on 31 January 2020). 
3. World-Health-Organization Statement on the second meeting of the International Health Regulations (2005) Emergency Committee regarding the outbreak of novel coronavirus (2019-nCoV). Available online: https://www.who.int/news-room/detail/30-01-2020-statement-on-the-second-meeting-ofthe-international-health-regulations-(2005)-emergency-committee-regarding-the-outbreak-of-novelcoronavirus-(2019-ncov) (accessed on 31 January 2020).

4. World-Health-Organization Coronavirus disease (COVID-19) outbreak. Available online: https://www.who. int/emergencies/diseases/novel-coronavirus-2019 (accessed on 31 January 2020).

5. World-Health-Organization Statement on the meeting of the International Health Regulations (2005) Emergency Committee regarding the outbreak of novel coronavirus (2019-nCoV). Available online: https://www.who.int/news-room/detail/23-01-2020-statement-on-the-meeting-of-the-international-healthregulations-(2005)-emergency-committee-regarding-the-outbreak-of-novel-coronavirus-(2019-ncov) (accessed on 31 January 2020).

6. Huang, C.; Wang, Y.; Li, X.; Ren, L.; Zhao, J.; Hu, Y.; Zhang, L.; Fan, G.; Xu, J.; Gu, X.; et al. Clinical features of patients infected with 2019 novel coronavirus in Wuhan, China. Lancet 2020, 395, 497-506. [CrossRef]

7. Heymann, D.L. Data sharing and outbreaks: Best practice exemplified. Lancet 2020, 395, 469-470. [CrossRef]

8. Liu, X.; Wang, X.-J. Potential inhibitors for 2019-nCoV coronavirus M protease from clinically approved medicines. bioRxiv 2020, 2020.01.29.924100.

9. Zhou, P.; Yang, X.-L.; Wang, X.-G.; Hu, B.; Zhang, L.; Zhang, W.; Si, H.-R.; Zhu, Y.; Li, B.; Huang, C.-L.; et al. A pneumonia outbreak associated with a new coronavirus of probable bat origin. Nature 2020. [CrossRef] [PubMed]

10. World-Health-Organization Update 49 - SARS case fatality ratio, incubation period. Available online: https://www.who.int/csr/sars/archive/2003_05_07a/en/ (accessed on 31 January 2020).

11. World-Health-Organization Middle East respiratory syndrome coronavirus (MERS-CoV). Available online: https://www.who.int/emergencies/mers-cov/en/ (accessed on 31 January 2020).

12. Lu, R.; Zhao, X.; Li, J.; Niu, P.; Yang, B.; Wu, H.; Wang, W.; Song, H.; Huang, B.; Zhu, N.; et al. Genomic characterisation and epidemiology of 2019 novel coronavirus: Implications for virus origins and receptor binding. Lancet 2020, 6736, 1-10. [CrossRef]

13. Letko, M.; Munster, V. Functional assessment of cell entry and receptor usage for lineage B $\beta$-coronaviruses, including 2019-nCoV. bioRxiv 2020, 2020.01.22.915660.

14. Hoffmann, M.; Kleine-Weber, H.; Kruger, N.; Muller, M.; Drosten, C.; Pohlmann, S. The novel coronavirus 2019 (2019-nCoV) uses the SARS-coronavirus receptor ACE2 and the cellular protease TMPRSS2 for entry into target cells. bioRxiv 2020, 2020.01.31.929042.

15. Yang, Z.-Y.; Kong, W.-P.; Huang, Y.; Roberts, A.; Murphy, B.R.; Subbarao, K.; Nabel, G.J. A DNA vaccine induces SARS coronavirus neutralization and protective immunity in mice. Nature 2004, 428, 561-564. [CrossRef]

16. Deming, D.; Sheahan, T.; Heise, M.; Yount, B.; Davis, N.; Sims, A.; Suthar, M.; Harkema, J.; Whitmore, A.; Pickles, R.; et al. Vaccine efficacy in senescent mice challenged with recombinant SARS-CoV bearing epidemic and zoonotic spike variants. PLoS Med. 2006, 3, e525. [CrossRef]

17. Graham, R.L.; Becker, M.M.; Eckerle, L.D.; Bolles, M.; Denison, M.R.; Baric, R.S. A live, impaired-fidelity coronavirus vaccine protects in an aged, immunocompromised mouse model of lethal disease. Nat. Med. 2012, 18, 1820-1826. [CrossRef] [PubMed]

18. Lin, Y.; Shen, X.; Yang, R.F.; Li, Y.X.; Ji, Y.Y.; He, Y.Y.; De Shi, M.; Lu, W.; Shi, T.L.; Wang, J.; et al. Identification of an epitope of SARS-coronavirus nucleocapsid protein. Cell Res. 2003, 13, 141-145. [CrossRef] [PubMed]

19. Wang, J.; Wen, J.; Li, J.; Yin, J.; Zhu, Q.; Wang, H.; Yang, Y.; Qin, E.; You, B.; Li, W.; et al. Assessment of immunoreactive synthetic peptides from the structural proteins of severe acute respiratory syndrome coronavirus. Clin. Chem. 2003, 49, 1989-1996. [CrossRef] [PubMed]

20. Liu, X.; Shi, Y.; Li, P.; Li, L.; Yi, Y.; Ma, Q.; Cao, C. Profile of antibodies to the nucleocapsid protein of the severe acute respiratory syndrome (SARS)-associated coronavirus in probable SARS patients. Clin. Vaccine Immunol. 2004, 11, 227-228. [CrossRef] [PubMed]

21. Tang, F.; Quan, Y.; Xin, Z.-T.; Wrammert, J.; Ma, M.-J.; Lv, H.; Wang, T.-B.; Yang, H.; Richardus, J.H.; Liu, W.; et al. Lack of peripheral memory B cell responses in recovered patients with severe acute respiratory syndrome: A six-year follow-up study. J. Immunol. 2011, 186, 7264-7268. [CrossRef] 
22. Peng, H.; Yang, L.-T.; Wang, L.-Y.; Li, J.; Huang, J.; Lu, Z.-Q.; Koup, R.A.; Bailer, R.T.; Wu, C.-Y. Long-lived memory $\mathrm{T}$ lymphocyte responses against SARS coronavirus nucleocapsid protein in SARS-recovered patients. Virology 2006, 351, 466-475. [CrossRef]

23. Fan, Y.-Y.; Huang, Z.-T.; Li, L.; Wu, M.-H.; Yu, T.; Koup, R.A.; Bailer, R.T.; Wu, C.-Y. Characterization of SARS-CoV-specific memory T cells from recovered individuals 4 years after infection. Arch. Virol. 2009, 154, 1093-1099. [CrossRef]

24. Ng, O.-W.; Chia, A.; Tan, A.T.; Jadi, R.S.; Leong, H.N.; Bertoletti, A.; Tan, Y.-J. Memory T cell responses targeting the SARS coronavirus persist up to 11 years post-infection. Vaccine 2016, 34, 2008-2014. [CrossRef]

25. Liu, W.J.; Zhao, M.; Liu, K.; Xu, K.; Wong, G.; Tan, W.; Gao, G.F. T-cell immunity of SARS-CoV: Implications for vaccine development against MERS-CoV. Antiviral Res. 2017, 137, 82-92. [CrossRef]

26. Li, C.K.-F.; Wu, H.; Yan, H.; Ma, S.; Wang, L.; Zhang, M.; Tang, X.; Temperton, N.J.; Weiss, R.A.; Brenchley, J.M.; et al. T cell responses to whole SARS coronavirus in humans. J. Immunol. 2008, 181, 5490-5500. [CrossRef]

27. Channappanavar, R.; Fett, C.; Zhao, J.; Meyerholz, D.K.; Perlman, S. Virus-specific memory CD8 T cells provide substantial protection from lethal severe acute respiratory syndrome coronavirus infection. J. Virol. 2014, 88, 11034-11044. [CrossRef] [PubMed]

28. Katoh, K.; Standley, D.M. MAFFT multiple sequence alignment software version 7: Improvements in performance and usability. Mol. Biol. Evol. 2013, 30, 772-780. [CrossRef] [PubMed]

29. Pickett, B.E.; Sadat, E.L.; Zhang, Y.; Noronha, J.M.; Squires, R.B.; Hunt, V.; Liu, M.; Kumar, S.; Zaremba, S.; $\mathrm{Gu}, \mathrm{Z}$; et al. ViPR: An open bioinformatics database and analysis resource for virology research. Nucleic Acids Res. 2012, 40, D593-D598. [CrossRef] [PubMed]

30. Vita, R.; Mahajan, S.; Overton, J.A.; Dhanda, S.K.; Martini, S.; Cantrell, J.R.; Wheeler, D.K.; Sette, A.; Peters, B. The immune epitope database (IEDB): 2018 update. Nucleic Acids Res. 2019, 47, D339-D343. [CrossRef]

31. Mirarab, S.; Nguyen, N.; Guo, S.; Wang, L.-S.; Kim, J.; Warnow, T. PASTA: Ultra-large multiple sequence alignment for nucleotide and amino-acid sequences. J. Comput. Biol. 2015, 22, 377-386. [CrossRef]

32. Huson, D.H.; Scornavacca, C. Dendroscope 3: An interactive tool for rooted phylogenetic trees and networks. Syst. Biol. 2012, 61, 1061-1067. [CrossRef]

33. Ahmed, S.F. Data and software code for reproducing results of this paper. Available online: https: //github.com/faraz107/2019-nCoV-T-Cell-Vaccine-Candidates (accessed on 31 January 2020).

34. Li, F. Structure of SARS coronavirus spike receptor-binding domain complexed with receptor. Science. 2005, 309, 1864-1868. [CrossRef]

35. Dahirel, V.; Shekhar, K.; Pereyra, F.; Miura, T.; Artyomov, M.; Talsania, S.; Allen, T.M.; Altfeld, M.; Carrington, M.; Irvine, D.J.; et al. Coordinate linkage of HIV evolution reveals regions of immunological vulnerability. Proc. Natl. Acad. Sci. 2011, 108, 11530-11535. [CrossRef]

36. Quadeer, A.A.; Louie, R.H.Y.; Shekhar, K.; Chakraborty, A.K.; Hsing, I.-M.; McKay, M.R. Statistical linkage analysis of substitutions in patient-derived sequences of genotype 1a hepatitis $C$ virus nonstructural protein 3 exposes targets for immunogen design. J. Virol. 2014, 88, 7628-7644. [CrossRef]

37. Ahmed, S.F.; Quadeer, A.A.; Morales-Jimenez, D.; McKay, M.R. Sub-dominant principal components inform new vaccine targets for HIV Gag. Bioinformatics 2019, 35, 3884-3889. [CrossRef]

38. Quadeer, A.A.; Morales-Jimenez, D.; McKay, M.R. Co-evolution networks of HIV/HCV are modular with direct association to structure and function. PLOS Comput. Biol. 2018, 14, e1006409. [CrossRef]

39. Prabakaran, P.; Gan, J.; Feng, Y.; Zhu, Z.; Choudhry, V.; Xiao, X.; Ji, X.; Dimitrov, D.S. Structure of severe acute respiratory syndrome coronavirus receptor-binding domain complexed with neutralizing antibody. $J$. Biol. Chem. 2006, 281, 15829-15836. [CrossRef] [PubMed]

40. Zhu, Z.; Chakraborti, S.; He, Y.; Roberts, A.; Sheahan, T.; Xiao, X.; Hensley, L.E.; Prabakaran, P.; Rockx, B.; Sidorov, I.A.; et al. Potent cross-reactive neutralization of SARS coronavirus isolates by human monoclonal antibodies. Proc. Natl. Acad. Sci. 2007, 104, 12123-12128. [CrossRef]

41. Hwang, W.C.; Lin, Y.; Santelli, E.; Sui, J.; Jaroszewski, L.; Stec, B.; Farzan, M.; Marasco, W.A.; Liddington, R.C. Structural basis of neutralization by a human anti-severe acute respiratory syndrome spike protein antibody, 80R. J. Biol. Chem. 2006, 281, 34610-34616. [CrossRef] [PubMed]

42. UniProt UniProtKB - P59594 (SPIKE_CVHSA). Available online: https://www.uniprot.org/uniprot/P59594 (accessed on 31 January 2020).

43. Walls, A.C.; Park, Y.-J.; Tortorici, M.A.; Wall, A.; McGuire, A.T.; Veesler, D. Structure, function and antigenicity of the SARS-CoV-2 spike glycoprotein. bioRxiv 2020, 2020.02.19.956581. 
44. Walls, A.C.; Xiong, X.; Park, Y.-J.; Tortorici, M.A.; Snijder, J.; Quispe, J.; Cameroni, E.; Gopal, R.; Dai, M.; Lanzavecchia, A.; et al. Unexpected receptor functional mimicry elucidates activation of coronavirus fusion. Cell 2019, 176, 1026-1039.e15. [CrossRef] [PubMed]

45. Walls, A.C.; Tortorici, M.A.; Snijder, J.; Xiong, X.; Bosch, B.-J.; Rey, F.A.; Veesler, D. Tectonic conformational changes of a coronavirus spike glycoprotein promote membrane fusion. Proc. Natl. Acad. Sci. 2017, 114, 11157-11162. [CrossRef] [PubMed]

46. Song, W.; Gui, M.; Wang, X.; Xiang, Y. Cryo-EM structure of the SARS coronavirus spike glycoprotein in complex with its host cell receptor ACE2. PLOS Pathog. 2018, 14, e1007236. [CrossRef]

47. Wrapp, D.; Wang, N.; Corbett, K.S.; Goldsmith, J.A.; Hsieh, C.-L.; Abiona, O.; Graham, B.S.; McLellan, J.S. Cryo-EM structure of the 2019-nCoV spike in the prefusion conformation. Science. 2020, 2011, eabb2507. [CrossRef]

48. Tian, X.; Li, C.; Huang, A.; Xia, S.; Lu, S.; Shi, Z.; Lu, L.; Jiang, S.; Yang, Z.; Wu, Y.; et al. Potent binding of 2019 novel coronavirus spike protein by a SARS coronavirus-specific human monoclonal antibody. Emerg. Microbes Infect. 2020, 9, 382-385. [CrossRef]

49. Ferguson, A.L.; Mann, J.K.; Omarjee, S.; Ndung'u, T.; Walker, B.D.; Chakraborty, A.K. Translating HIV sequences into quantitative fitness landscapes predicts viral vulnerabilities for rational immunogen design. Immunity 2013, 38, 606-617. [CrossRef] [PubMed]

50. Chakraborty, A.K.; Barton, J.P. Rational design of vaccine targets and strategies for HIV: A crossroad of statistical physics, biology, and medicine. Reports Prog. Phys. 2017, 80, 032601. [CrossRef] [PubMed]

51. Quadeer, A.A.; Louie, R.H.Y.; McKay, M.R. Identifying immunologically-vulnerable regions of the HCV E2 glycoprotein and broadly neutralizing antibodies that target them. Nat. Commun. 2019, 10, 2073. [CrossRef] [PubMed]

52. Louie, R.H.Y.; Kaczorowski, K.J.; Barton, J.P.; Chakraborty, A.K.; McKay, M.R. Fitness landscape of the human immunodeficiency virus envelope protein that is targeted by antibodies. Proc. Natl. Acad. Sci. 2018, 115, E564-E573. [CrossRef]

53. Quadeer, A.A.; Barton, J.P.; Chakraborty, A.K.; McKay, M.R. Deconvolving mutational patterns of poliovirus outbreaks reveals its intrinsic fitness landscape. Nat. Commun. 2020, 11, 377. [CrossRef]

54. Mann, J.K.; Barton, J.P.; Ferguson, A.L.; Omarjee, S.; Walker, B.D.; Chakraborty, A.; Ndung'u, T. The fitness landscape of HIV-1 Gag: Advanced modeling approaches and validation of model predictions by in vitro testing. PLoS Comput. Biol. 2014, 10, e1003776. [CrossRef]

55. Ramaiah, A.; Arumugaswami, V. Insights into cross-species evolution of novel human coronavirus 2019-nCoV and defining immune determinants for vaccine development. bioRxiv 2020, 2020.01.29.925867. 


\section{University Library}

\section{- MINERVA \\ A gateway to Melbourne's research publications}

Minerva Access is the Institutional Repository of The University of Melbourne

Author/s:

Ahmed, SF;Quadeer, AA;McKay, MR

Title:

Preliminary Identification of Potential Vaccine Targets for the COVID-19 Coronavirus (SARSCoV-2) Based on SARS-CoV Immunological Studies

Date:

2020-03-01

\section{Citation:}

Ahmed, S. F., Quadeer, A. A. \& McKay, M. R. (2020). Preliminary Identification of Potential Vaccine Targets for the COVID-19 Coronavirus (SARS-CoV-2) Based on SARS-CoV Immunological Studies. VIRUSES-BASEL, 12 (3), https://doi.org/10.3390/v12030254.

Persistent Link:

http://hdl.handle.net/11343/296039

License:

CC BY 